Thorax (1969), 24, 435.

\title{
Excision of oesophageal varices
}

\author{
J.B. SELF \\ From Leicester General Hospital
}

Treatment of bleeding oesophageal varices by total excision of the stomach and oesophagus up to the aortic arch is suggested when a shunt is impossible or contra-indicated. The technique is described and six cases are presented.

After treating bleeding oesophageal varices for a number of years, mainly by porta caval shunts with oesophageal and gastric transections as a second line of treatment, it was realized that the fact that varices in the intestinal tract are localized could be made use of in their surgical treatment. Bleeding from the rectum in portal hypertension is rare and it never seems to occur from the small bowel. It would appear that the varices that bleed are confined to the oesophagus below the arch of the aorta and the stomach and occasionally the rectum (Fig. 1).

An effective shunt should lower the portal pressure, stop an acute haemorrhage, and have con-

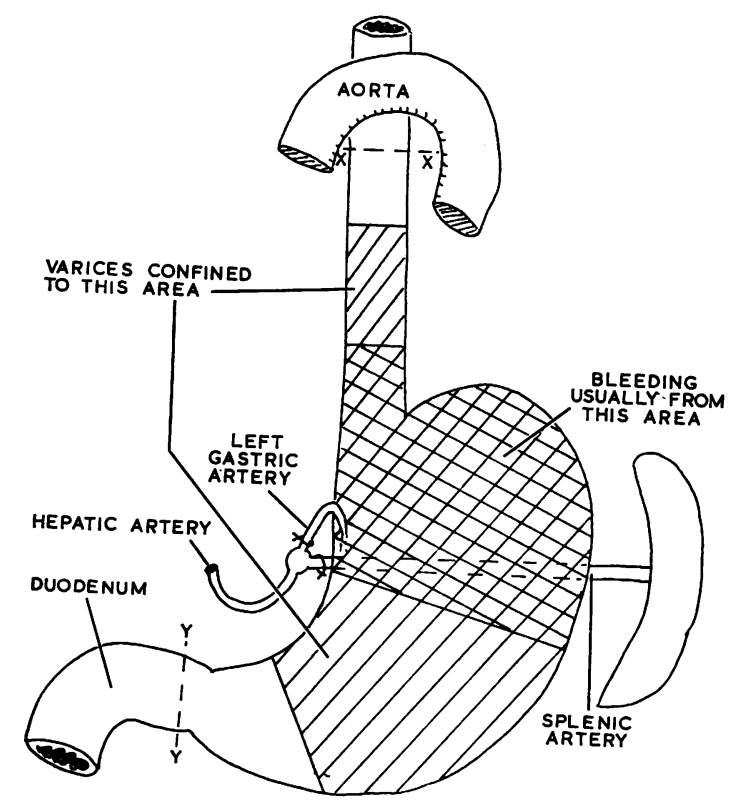

FIG. 1. Diagram to show extent of resection $X Y$. siderable success in preventing recurrent haemorrhage from varices. If, however, a shunt is thrombosed or for some reason impossible to construct, oesophageal transection or injection of the varices may be resorted to. Unfortunately, although effective initially, haemorrhage frequently recurs in two or three years' time.

A resection of the whole varix-bearing area (Fig. 1) has been adopted over the past few years in patients with thrombosed portal veins, especially when the splenic vein is also occluded. As the spleen is removed and the splenic and left gastric arteries are divided, a considerable reduction of the inflow of blood to the portal system is also secured. The resected portion of gut is replaced with an isolated loop of jejunum. Veins in the jejunum, although enlarged in portal hypertension, do not form submucosal varices and would seem unlikely to bleed: furthermore, the veins do not run far longitudinally in the bowel wall and it is hoped that they are therefore unlikely to provide a large flow to the oesophageal veins at the level of the aortic arch where the anastomosis is constructed. Should anastomotic channels develop between the veins in the mesentery of the loop and the mediastinal veins, this should provide a beneficial rather than a harmful shunt.

The patient is placed in the left lateral position with the right knee flexed and the left leg extended. The left hip is rotated back to provide a semi-supine position for the abdomen while the thorax remains in a true lateral position. This provides optimum access to the upper abdomen and also to the chest. A long abdomino-thoracic incision along the eighth rib is used extending just across the mid-line above the umbilicus. The diaphragm is detached circumferentially from its attachment to the chest wall above and below the incision. With a retractor in position a wide view of the whole operative field is obtained.

The oesophagus is mobilized. The pleura over the 


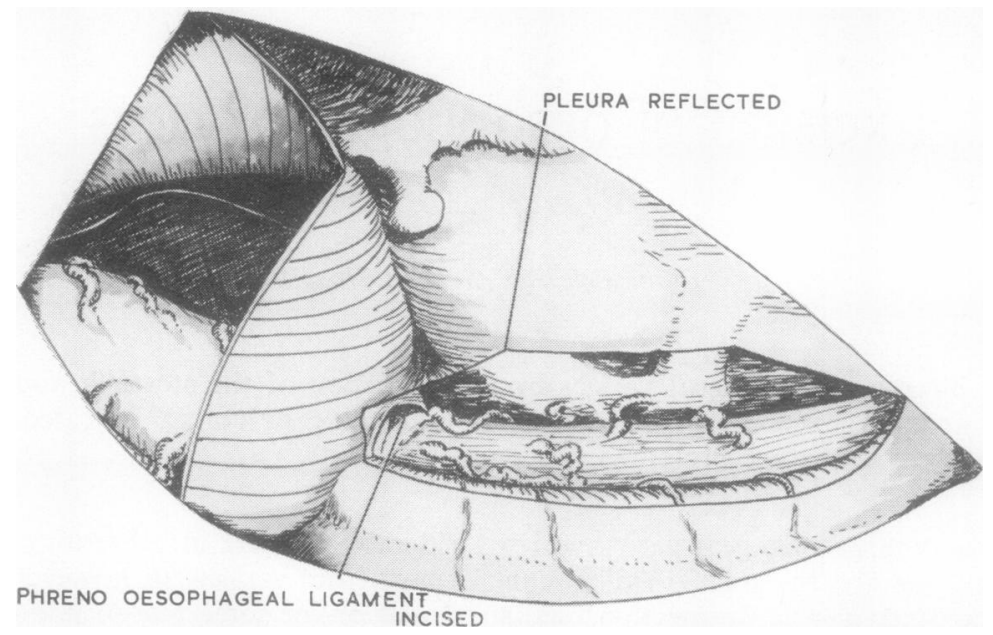

FIG. 2. Pleura incised and dissected off the oesophagus and phreno-oesophageal ligament incised.

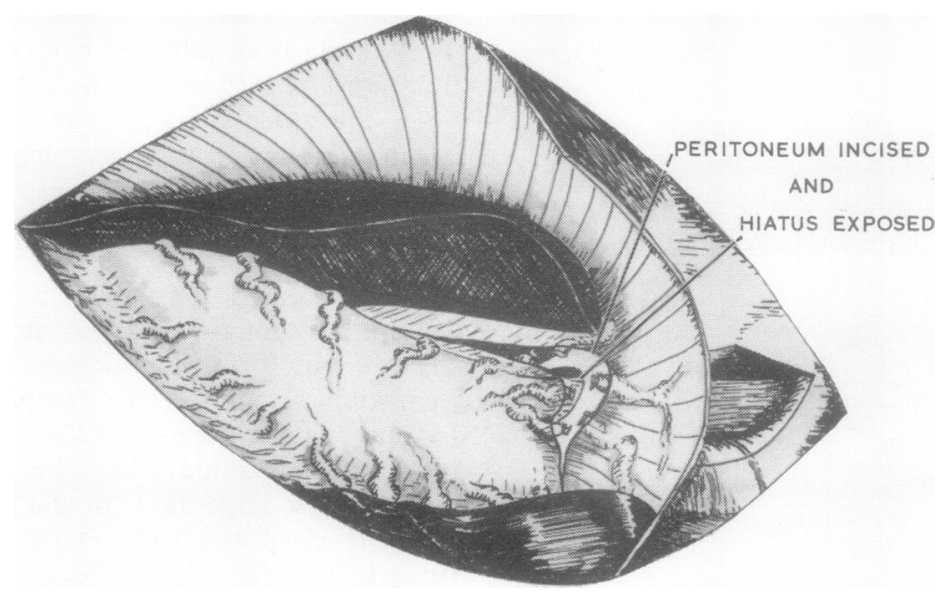

FIG. 3. The initial dissection around the hiatus below the diaphragm.

oesophagus is incised from the diaphragm up on to the arch of the aorta and the oesophagus is exposed with all the enlarged veins running from it to the mediastinum (Fig. 2). Each vein is doubly ligated and divided. The dissection round the oesophagus is continued, including division of the oesophageal arteries, until the oesophagus is completely freed from the diaphragm to the aortic arch. The margins of the hiatus are cleared and defined as much as possible from the thoracic side. The dissection is then continued below the diaphragm. The peritoneum is incised on the diaphragm around the hiatal margin (Fig. 3) and the numerous large veins passing from the stomach to the diaphragm are doubly ligated and divided. This incision and dissection is carried down, first on the right to the gastro-hepatic omentum and then rounch the fundus of the stomach to the left on to the lienoo renal ligament. The spleen is mobilized and the splenic artery and all the large veins are ligated and divided. This part of the dissection can be difficulto After mobilizing the spleen the greater curvature of the stomach is mobilized by division of the greatep omentum outside the gastro-epiploic arcade, con:tinuing the dissection until the right gastro-epiploif vessels are divided and the lower border of the firs part of the duodenum is cleared. The spleen and stomach are now retracted to the right and the lef gastric vessels are identified, ligated, and dividedo Numerous large venous channels are found at this point between the posterior wall of the stomach an 


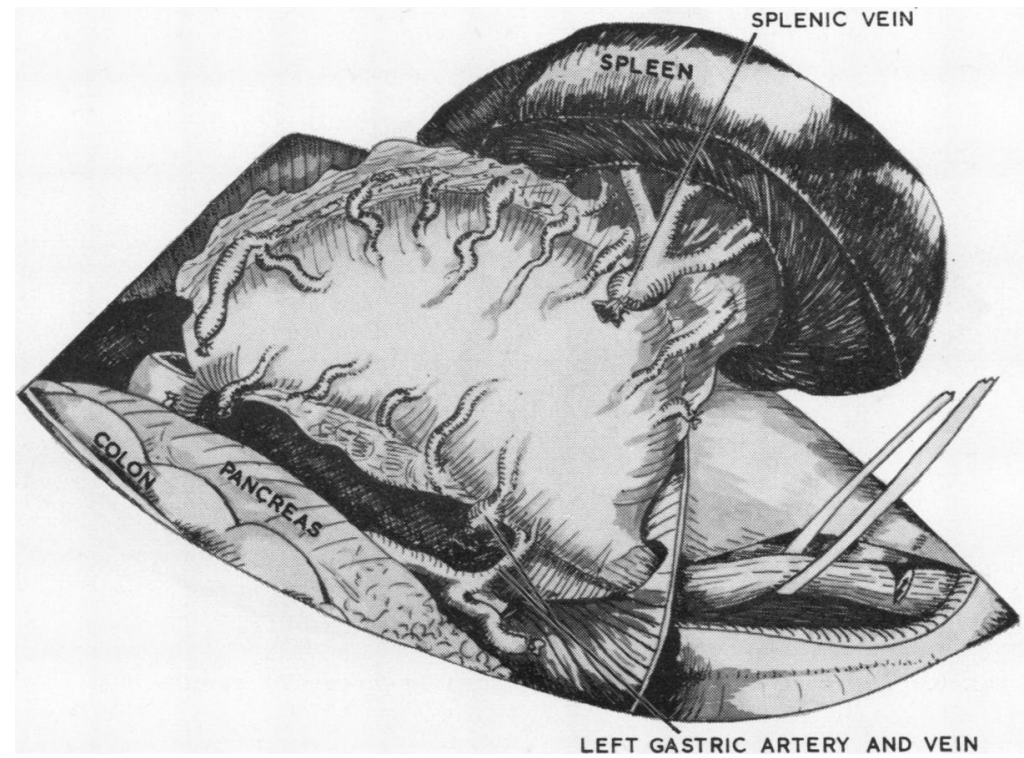

FIG. 4. The oesophagus fully mobilized, and mobilization of the spleen and stomach almost completed.

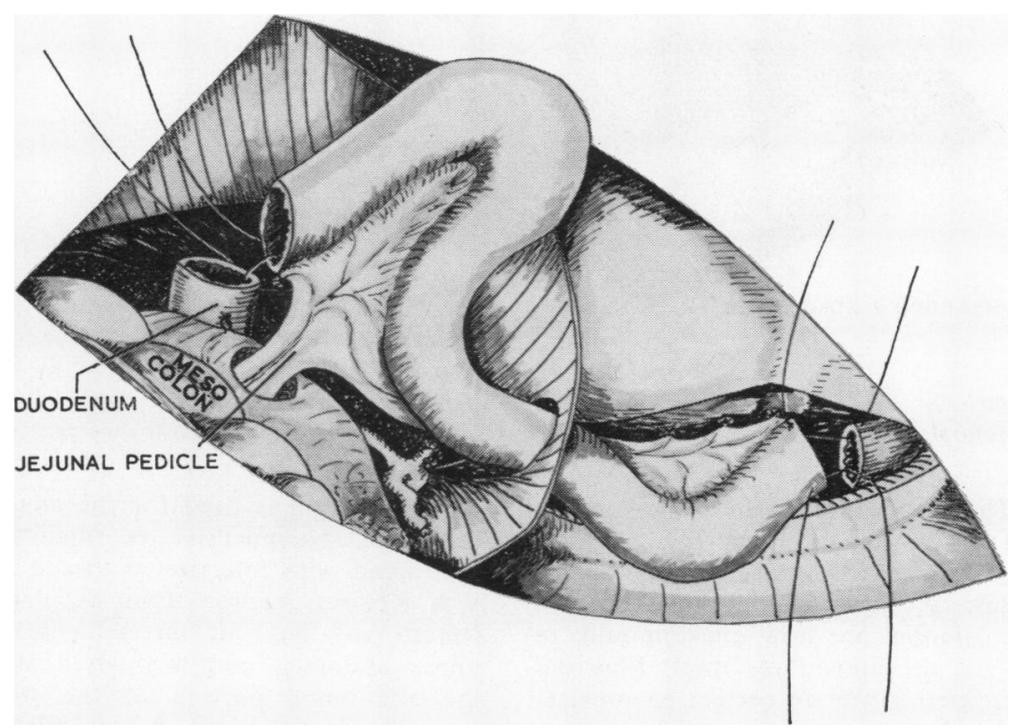

FIG. 5. Jejunal loop in place ready for anastomosis.

the posterior abdominal wall and have to be divided and ligated one by one. The remainder of the lesser omentum is then divided, including the right gastric vessels, and the upper border of the duodenum is cleared (Fig. 4). All remaining tissue around the abdominal oesophagus is cleared, completing the mobilization. A loop of jejunum of a sufficient length to reach as far as the arch of the aorta is now isolated. The oesophagus is clamped and divided just below the arch of the aorta and the duodenum is divided. The jejunal loop is then anastomosed to the oesophagus end to end (Fig. 5) using a single layer of interrupted linen thread sutures through all layers, taking special care to include a good bite of 


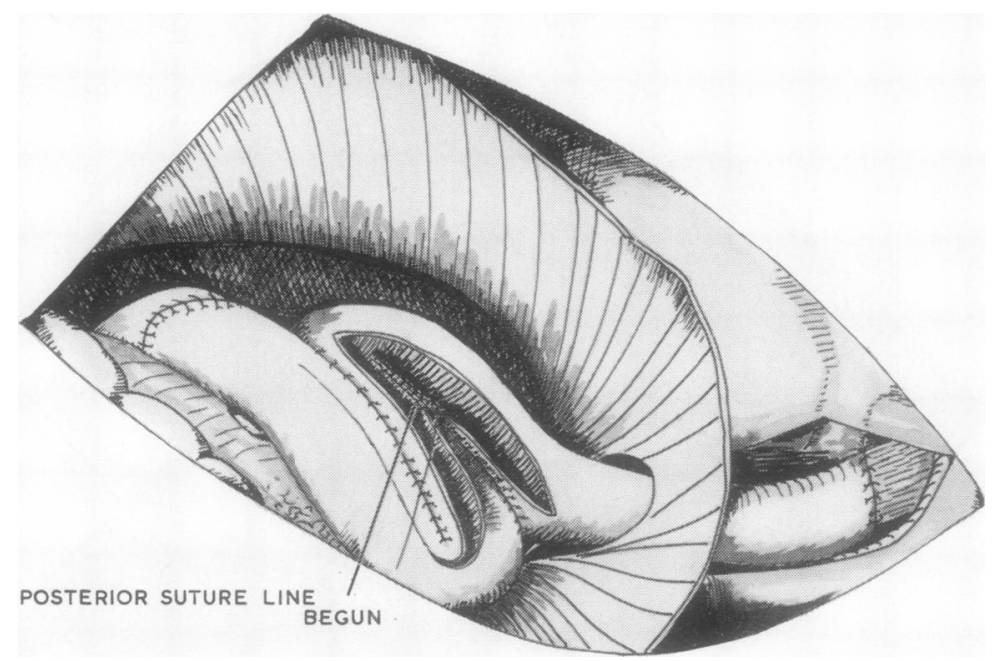

FIG. 6. The triple jejunal loop anastomosis in process of construction.

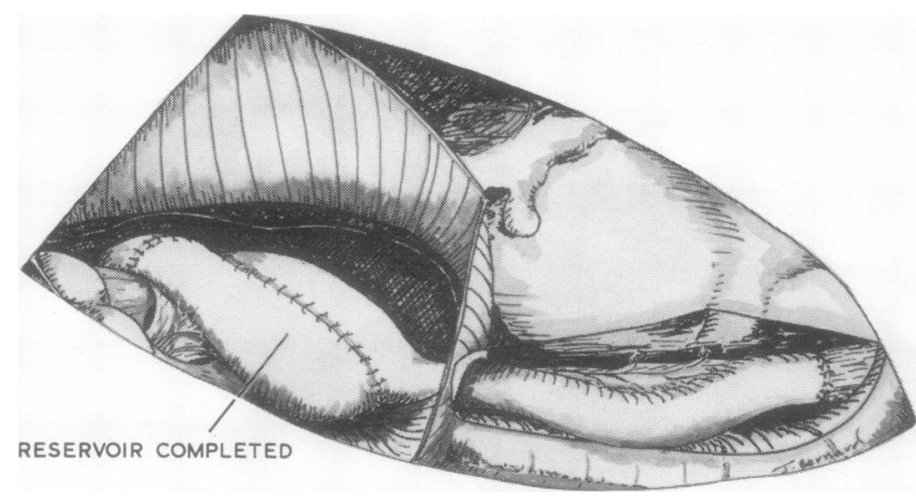

FIG. 7. The anastomoses completed prior to closing the pleura and inserting drains.

the submucosa. The duodenum is then anastomosed to the lower end of the jejunal loop (Fig. 5), again using one layer of interrupted sutures, after securing complete haemostasis of the intestinal ends. The two loose ends of the jejunum are now anastomosed, restoring continuity of the alimentary tract. Fine diathermy forceps are used to obtain perfect haemostasis - before using a single layer of interrupted suturesfor all these intestinal anastomoses which otherwise are very bloody. The sub-diaphragmatic portion of the long jejunal loop is then converted into a reservoir using a three-loop jejunal pouch (Figs 6 and 7). The long S-shaped anastomosis needs to be made with care, because in portal hypertension the jejunum is congested. The incision is made with a fine diathermy knife and diathermy forceps are used to obtain haemostasis. A layer of continuous 00 chromic catgut is used for the anastomosis to ensur? that the haemostasis is maintained and it is reinforced with interrupted thread sutures.

A Redivac suction drain is placed in the mediaA stinum and led out through the hiatus across the upper abdomen and through a stab incision below the abdominal portion of the wound. The pleura over the mediastinum is carefully closed with fine interrupted thread sutures. Another suction drain is placed in the gastro-splenic bed and led out through ${ }^{+}$ a separate stab incision. The diaphragm is reattached to the chest wall with interrupted thread sutures. chest drain with underwater seal is used through stab incision in the tenth intercostal space in thळ posterior axillary line. The whole wound is then closed in layers.

Routine post-operative care is given, the ches 
drain being withdrawn after 48 hours. Intravenous fluids are maintained for four days, the patient having nothing by mouth for 48 hours. Sips of water are then allowed. Free fluids and semi-solids are given on the fifth day and the diet is gradually increased until a normal diet is being taken by the eighth to tenth day. The drain to the splenic bed is removed on the fifth day and that in the mediastinum on the seventh day. The patient has injections of cyanocobalamin, $1,000 \mu \mathrm{g}$. , once a month.

This operation has now been used for the past four years in patients who have thrombosed portal veins and who for various reasons have been found unsuitable for spleno-renal shunts. Most have been operated on while bleeding when medical measures have failed to arrest the haemorrhage. One patient was known to have had a very poor hepatic function for over a year and it was thought that a portacaval shunt would almost certainly have tipped her into complete hepatic failure. Therefore although the portal vein was patent a resection was carried out instead.

\section{CASE REPORTS}

CASE 1 A young woman aged 28 was seen on 18 December 1964 with uncontrolled bleeding from oesophageal varices. She had a long history. Her first haematemesis was in 1953 at the age of 17. She was treated for one year as having a bleeding peptic ulcer, and was then diagnosed as having Banti's disease. In 1955 she had a laparotomy and splenectomy. After further bleeding she had the oesophageal varices injected. In 1957 she had a porta-caval shunt. She had a relapse following this and then had an oesophago-gastric transection. She was well for one year and then haematemeses started again. In 1960 she moved to Leicester and was admitted with haematemesis in January 1960 and again in March. A laparotomy on 28 March 1960 showed a thrombosed porta-caval shunt and a venogram showed no splenic vein filling; a further gastric transection was carried out. In June 1961 she had a further melaena. She then remained well until her admission in December 1964. On this occasion a Sengstaken tube failed to control the bleeding and she was referred for surgery. The prospect of any surgery being successful seemed remote, but as a last desperate measure it was decided, if technically possible, to resect the whole varix-bearing area. On 18 December 1964 the old left thoracotomy scar was reopened. The pleural cavity and upper abdominal cavity were obliterated with adhesions full of large anastomotic veins making the dissection difficult. The whole stomach and 5 in. $(12.7 \mathrm{~cm}$.) of the oesophagus were resected and replaced with a reconstruction using a jejunal loop. She made an uneventful recovery. Her weight which had been 8 st. $6 \mathrm{lb}$. $(53.5 \mathrm{~kg}$.) for some years rose to 9 st. $2 \mathrm{lb}$. $(58 \cdot 0 \mathrm{~kg}$.) by April 1965 . Her haemoglobin has not fallen below $90 \%$ since her recovery although it had not been recorded as high as $90 \%$ during the previous three years. Her liver function tests have always been within normal limits. She remains well four years later, and eats bigger meals than for some years before this last operation.

CASE 2 A young man aged 21 was seen in April 1963 with a long history of haematemeses since 1952 . Oesophageal varices had been diagnosed and he had been treated conservatively until March 1953, when a thoraco-abdominal exploration revealed a splenic vein thrombosis and a portal thrombosis. On 24 April 1963 a spleno-portagram showed a patent splenic vein and what appeared to be a patent portal vein. A porta-caval shunt therefore seemed a possibility. On 3 May 1963 a laparotomy showed a normal liver, massive intraperitoneal adhesions, and the portal vein replaced by a plexus of thin-walled veins. The splenic vein was also plexiform. An anastomosis was impossible. In October 1964 he had a further haematemesis and again on 30 September 1966 he had a severe one. It was decided to attempt a resection of the varix-bearing area as an emergency. On 1 October 1966 the old thoraco-abdominal incision was reopened. Massive adhesions and large venous channels made the dissection difficult. A splenectomy was performed, the oesophagus up to the aortic arch and the stomach was resected. A jejunal loop was used to replace the stomach and lower oesophagus. He made a rapid recovery and has regained his pre-operative weight. His liver function tests at all times have remained within normal limits. He is now 1 st. $(6 \cdot 3 \mathrm{~kg}$.) heavier than he was in 1963.

CASE 3 A woman aged 49 was seen on 3 December 1965 with a haematemesis and a haemoglobin of $34 \%$. In December 1962 she had had peritonitis and jaundice. She remained slightly jaundiced. She was admitted with a haematemesis on 3 December 1965 and a barium meal showed varices. The question of exploring the portal vein with a view to a shunt was considered, but in view of her consistently poor liver function it was deferred and her haemorrhage settled. Haemorrhage, however, was recurrent, but it was feared that with a persistent degree of hepatic failure a shunt would almost certainly lead to complete hepatic failure. Excision of the varices was therefore carried out and a reconstruction using a jejunal loop was performed. She made an uneventful recovery without any evidence of increased hepatic failure. On 10 May 1967 she weighed the same as she had six months previously and a barium meal showed no varices; her liver function has not deteriorated at all since.

CASE 4 A woman aged 54 was seen in February 1967 with a massive haematemesis. She had had a number of haematemeses over the past two years from proved oesophageal varices. She had had 
ascending cholangitis in 1961. On two occasions she had developed hepatic coma, and once hypertensive cardiac failure. She was known to have hypertension and left bundle-branch block, but as bleeding continued despite insertion of a Sengstaken tube her portal vein was explored on 19 February 1967 although she had not had a portagram. The portal and splenic veins were found to be thrombosed and the peritoneal cavity was obliterated by adhesions. A resection of the stomach and oesophagus up to the aortic arch was carried out with jejunal replacement. Six days later she developed jaundice and early cardiac failure. The jaundice had cleared by the eleventh post-operative day, but she died of cardiac failure on the thirteenth post-operative day. Postmortem examination showed left ventricular hypertrophy, coronary atherosclerosis, and ischaemic myocardial fibrosis.

CASE 5 A young man aged 26 was seen in February 1968 with a massive haematemesis. He had a history of repeated haematemeses for four years of increasing severity. His liver function tests were always within normal limits, liver biopsy was normal, and a spleno-portagram showed a plexiform replacement of the portal and splenic veins. As bleeding continued resection of the stomach and oesophagus up to the aortic arch was performed as an emergency. Post-operatively he had a stormy course, developing an empyema. This was drained and he gradually improved until he had a pulmonary embolus one day while walking about in the ward and died within few minutes six weeks post-operatively.

CASE 6 A woman aged 43 was seen in April 196 having had a haematemesis uncontrolled by Sengstaken tube. She had had a splenectomy an $\$$ gastric transection 18 months previously. Liver biopsy had been normal and the portal vein had been found to be thrombosed. An emergency resection of the stomach and oesophagus up to the aortic arch was. performed. Post-operatively she made a satisfactory recovery, developing a transient ascites and suffering from some degree of dumping which has since settleok considerably. Her weight remains a few pounds below its normal level.

This technique is considered worth presentinf because five out of the six cases had uncontrolled bleeding with little prospect of controlling it per=manently with any other form of treatment. The mortality of the operation at $33 \%$ is high but the cases were desperate emergencies. Those who have survived have had gratifying results so faro None has bled again. Only one has had any் form of indigestion. All but one have at leasథ regained their pre-operative weight. Case 3 is interesting in that her hepatic failure was no increased by the operation, in contrast to manos patients having porta-caval shunts. Indeed onle one of the six cases (case 4) showed any hepatio failure as a result of the operation. 